\title{
. \\ Internal Medicine \\ Treatment with rivaroxaban and monitoring of coagulation profiles in two dogs with venous thromboembolism
}

\author{
Mona UCHIDA ${ }^{1)}$, Aki OHMI'), Reina FUJIWARA ${ }^{2)}$, Kenjiro FUKUSHIMA ${ }^{2)}$, \\ Akihiro DOI ${ }^{2)}$, Kazushi AZUMA ${ }^{2)}$ and Hajime TSUJIMOTO ${ }^{3) *}$ \\ 1)Department of Veterinary Clinical Pathobiology, Graduate School of Agricultural and Life Sciences, \\ The University of Tokyo, 1-1-1 Yayoi, Bunkyo-ku, Tokyo 113-8657, Japan \\ ${ }^{2)}$ Veterinary Medical Center, Graduate School of Agricultural and Life Sciences, The University of Tokyo, \\ 1-1-1 Yayoi, Bunkyo-ku, Tokyo 113-8657, Japan \\ ${ }^{3)}$ Department of Veterinary Internal Medicine, Graduate School of Agricultural and Life Sciences, \\ The University of Tokyo, 1-1-1 Yayoi, Bunkyo-ku, Tokyo 113-8657, Japan
}

\section{J. Vet. Med. Sci.}

82(9): 1271-1276, 2020

doi: 10.1292/jvms.19-0605

Received: 6 November 2019 Accepted: 30 June 2020 Advanced Epub:

13 July 2020

\begin{abstract}
Two dogs with immune-mediated hemolytic anemia complicated with thromboembolism were presented. Both of the dogs were initially treated with immunosuppressive therapy in conjunction with dalteparin and clopidogrel. Although the immunosuppressive therapy was effective, peritoneal effusion due to thromboembolism was observed during the course of the disease in these dogs. After initiation of rivaroxaban treatment, peritoneal effusion decreased immediately in parallel with the normalization of D-dimer, antithrombin (AT), and thrombinantithrombin complex (TAT). Hematochezia, cutaneous hemorrhage, and hematuria were observed as adverse events after administration of rivaroxaban in one case. Rivaroxaban was effective for the control of thromboembolism secondary to immune-mediated hemolytic anemia, and D-dimer, AT, and TAT were useful to monitor the status of thromboembolic disease in dogs.

KEY WORDS: direct oral anticoagulant, heparin resistance, immune-mediated hemolytic anemia, thrombotic marker, venous thromboembolism
\end{abstract}


count: $235,000 / \mu l$ ), and a blood smear showed the presence of spherocytosis and polychromasia. The platelet count was within the reference range $(414,000 / \mu l$; reference range, $148,000-484,000 / \mu l)$. The dog was diagnosed with primary IMHA based on exclusion of possible causes of secondary IMHA through history, physical examination, blood examination, thoracic radiography and abdominal ultrasound. Abdominal ultrasonography and a systemic computed tomography (CT) scan showed accumulation of peritoneal effusion in conjunction with the presence of a thrombus in the portal vein. To evaluate the coagulation state, TAT was measured using point-of-care analyzer, PATHFAST (LSI Medience Corporation, Tokyo, Japan), and D-dimer and AT were measured at a commercial laboratory, LSI Medience Corporation. Prothrombin time (PT) and activated partial thromboplastin time (aPTT) were measured by COAG1 (Wako Pure Chemical, Osaka, Japan). While there was no substantial prolongation of PT ( 8.1 sec; reference range, $6-9 \mathrm{sec})$ and aPTT $(19 \mathrm{sec}$; reference range, $11-18 \mathrm{sec})$, increased TAT concentration $(0.7 \mathrm{ng} / \mathrm{ml} ; \mathrm{reference}$ range $<0.2 \mathrm{ng} / \mathrm{m} l)$ and D-dimer concentration $(6.25 \mu \mathrm{g} / \mathrm{m} l$; reference range, $<1 \mu \mathrm{g} / \mathrm{m} l)$, and decreased AT activity $(37 \%$; reference range, 116-161\%) were observed, suggesting that the dog was complicated with thromboembolism associated with IMHA.

The dog was treated with immunosuppressive agents including prednisolone (Predonine; $2 \mathrm{mg} / \mathrm{kg}$, PO, q 24 hr; Shionogi, Osaka, Japan), mycophenolate mofetil (Cellcept; $15 \mathrm{mg} / \mathrm{kg}$, PO, q 24 hr; Chugai Pharmaceutical, Tokyo, Japan), and cyclosporine (Ciclosporin; 4 mg/kg, PO, q 24 hr; Nichi-Iko Pharmaceutical, Toyama, Japan) in conjunction with antithrombotic agents including dalteparin (Dalteparin Na; $150 \mathrm{U} / \mathrm{kg} /$ day constant rate infusion [CRI]; Nichi-Iko Pharmaceutical) and clopidogrel (Plavix; 3 mg/kg, PO, q 24 hr; Sanofi K. K., Tokyo, Japan). Ultrasonography on Day 17 revealed additional thrombus formation in the splenic vein (Fig. 1A) and reassessment of thromboembolism by a CT scan on Day 24 revealed thrombus formation in the splenic and caudal mesenteric veins in addition to the portal vein (Fig. 1B-D).

Since no improvements in the coagulation state was observed, fresh plasma transfusion $(10 \mathrm{~m} / \mathrm{kg})$ was performed because of the reduced AT activity on Days 18, 19, 26, and 27. However, values of AT, D-dimer and TAT were still outside of the reference range, and the thrombus size and accumulation of ascites remained unchanged. Because antithrombotic therapy with dalteparin and clopidogrel was supposed to be ineffective, dalteparin was replaced with rivaroxaban (Xarelto; $0.8 \mathrm{mg} / \mathrm{kg}, \mathrm{PO}, \mathrm{q} 24 \mathrm{hr}$; Bayer Pharma AG, Osaka, Japan) on Day 31. After initiation of rivaroxaban administration, the thrombus started to reduce in size and peritoneal effusion gradually decreased and completely resolved 14 days later.

Three days after administration of rivaroxaban, bleeding events such as cutaneous hemorrhage and hematochezia were observed, but they resolved several days after the reduction of its dose $(0.4 \mathrm{mg} / \mathrm{kg}, \mathrm{PO}, \mathrm{q} 24 \mathrm{hr})$. On Day 80, the dog showed hematuria, and the dose of rivaroxaban and clopidogrel were reduced to $0.4 \mathrm{mg} / \mathrm{kg} /$ day and $3 \mathrm{mg} / \mathrm{kg} /$ day, respectively, to prevent further hemorrhage. Hematuria resolved soon after dose reduction, suggesting that hematuria was associated with the influence by the antithrombotic agents including rivaroxaban. Slight prolongations of aPTT were observed on Day 31 (18.4 sec) and on Day 80 (20.9 sec) when bleeding events were observed. Moreover, the level of TAT increased on Day 80 and then reduced after the resolution of hematuria. The dog also showed temporary diarrhea and anorexia on Day 80 and the symptoms also resolved after dose reduction, but association between these clinical signs and antithrombotic therapy was unknown.

At the reexamination on Day 98, the thrombus in the splenic vein disappeared (Fig. 1E), and PCV and coagulation parameters
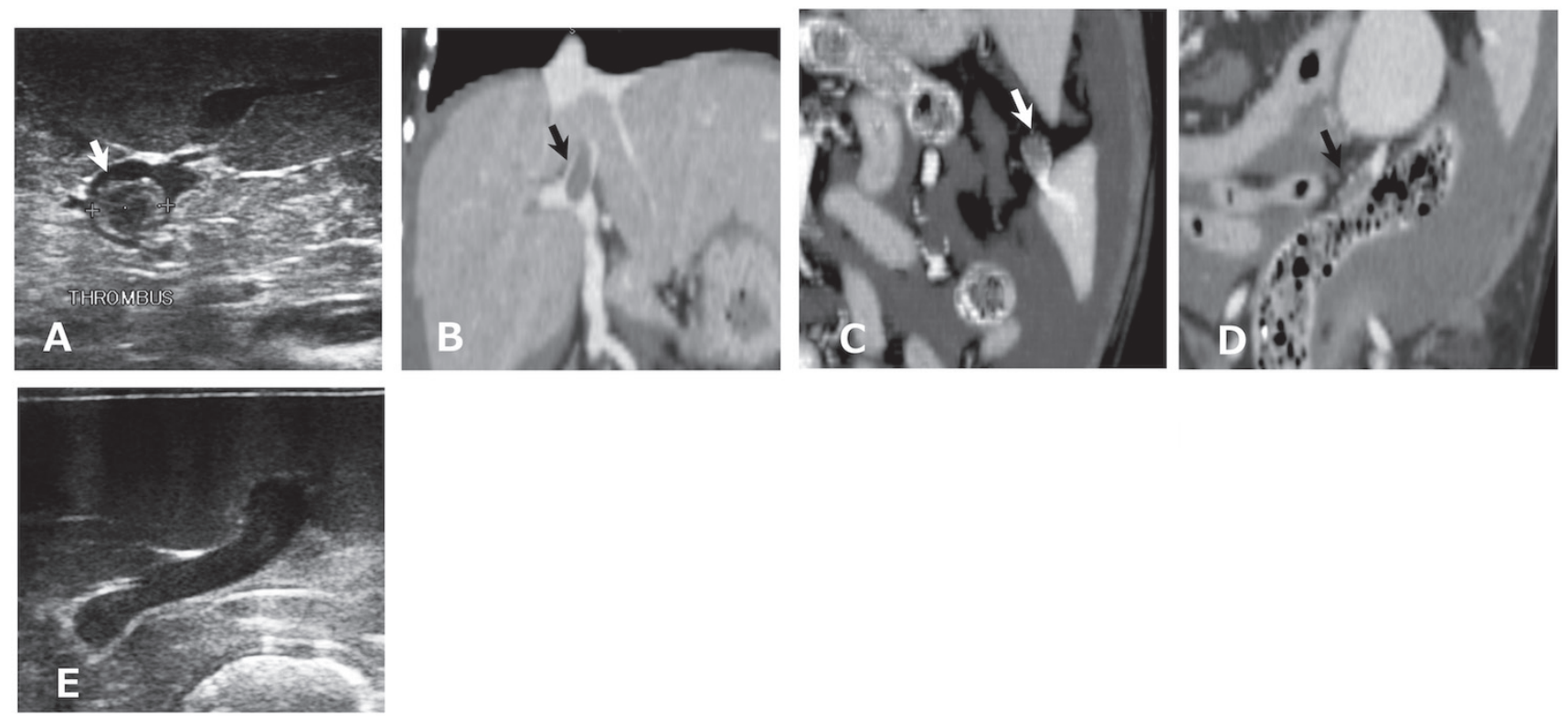

Fig. 1. Diagnostic imaging of thrombi in Dog 1. Ultrasonography of the splenic vein (A, E) and transverse image of the abdominal computed tomography (CT) (B, C, D). The thrombus (arrow) in the splenic vein was visualized by ultrasonography (A), but disappeared after treatment (E). In the abdominal CT scan in the post-contrast phase, thrombi (arrows) were found in the portal vein (B), the splenic vein (C), and the caudal mesenteric vein (D). 
normalized (Fig. 2). PT showed no notable prolongation throughout the course of the disease. Rivaroxaban was discontinued on Day 103 because ultrasonography revealed no thrombus and coagulation profiles remained normalized at this point. At the most recent follow-up, 430 days after cessation of rivaroxaban treatment, there was no relapse of IMHA or thromboembolism. The dog kept administration of clopidogrel up until the time of preparing this paper.

Dog 2: A 5-year-old spayed female Miniature Pinscher was referred to VMC of UTokyo for evaluation of lethargy and anorexia. CBC showed regenerative anemia (PCV: 13\%, reticulocytes: 277,000/ $\mu l$ ) and normal platelet count $(226,000 / \mu l)$. The dog was diagnosed with IMHA with the spherocytosis and a positive direct Coombs' test with an exclusion of other possible conditions associated with hemolytic anemia. To evaluate the coagulation state, the same methods were used to measure TAT, D-dimer and AT as used in Dog 1, and COAG2NV (Fujifilm Wako Pure Chemical, Osaka, Japan) was used to measure PT and aPTT. At the first presentation on Day 1, both PT and aPTT were in the reference ranges (PT, $7.7 \mathrm{sec}$; reference range, 6.8-8.6 sec) (aPTT, 22.2 sec; reference range, 13.1-26.9 sec). Meanwhile, decreased AT activity $(85 \%)$, increased D-dimer concentration $(5.5 \mu \mathrm{g} / \mathrm{m} l)$, and increased TAT concentration $(1.79 \mathrm{ng} / \mathrm{m} l)$ indicated the thrombotic tendency. Although platelet count was within the normal range, the dog was suspected to be in an early phase of disseminated intravascular coagulation (DIC) from the findings of elevated TAT in addition to abnormal values of D-dimer and AT activity $[9,26]$.

The dog was treated with prednisolone (Predonine; $2 \mathrm{mg} / \mathrm{kg}$, PO, q $12 \mathrm{hr}$; Shionogi), cyclosporine (Atopica; $5.5 \mathrm{mg} / \mathrm{kg}, \mathrm{PO}$, q 24 hr; Novartis Pharma K. K., Tokyo, Japan), dalteparin (Dalteparin Na; 100 U/kg/day CRI; Nichi-Iko Pharmaceutical), and clopidogrel (Plavix; 4.7 mg/kg, PO, q 24 hr; Sanofi K. K.) after administration of intravenous human immunoglobulin (IVIG) (Gammagard; 0.6 g/kg, CRI; Baxter Healthcare Corp., Deerfield, IL, USA) and a whole fresh blood transfusion (20 ml/kg) on Day 1 . The dog responded to the treatment; PCV increased to $21.4 \%$, TAT decreased to $0.352 \mathrm{ng} / \mathrm{ml}$, suggesting the recovery from IMHA and suspected DIC. However, accumulation of peritoneal effusion and edema in the hind limbs were noted on Day 5, and
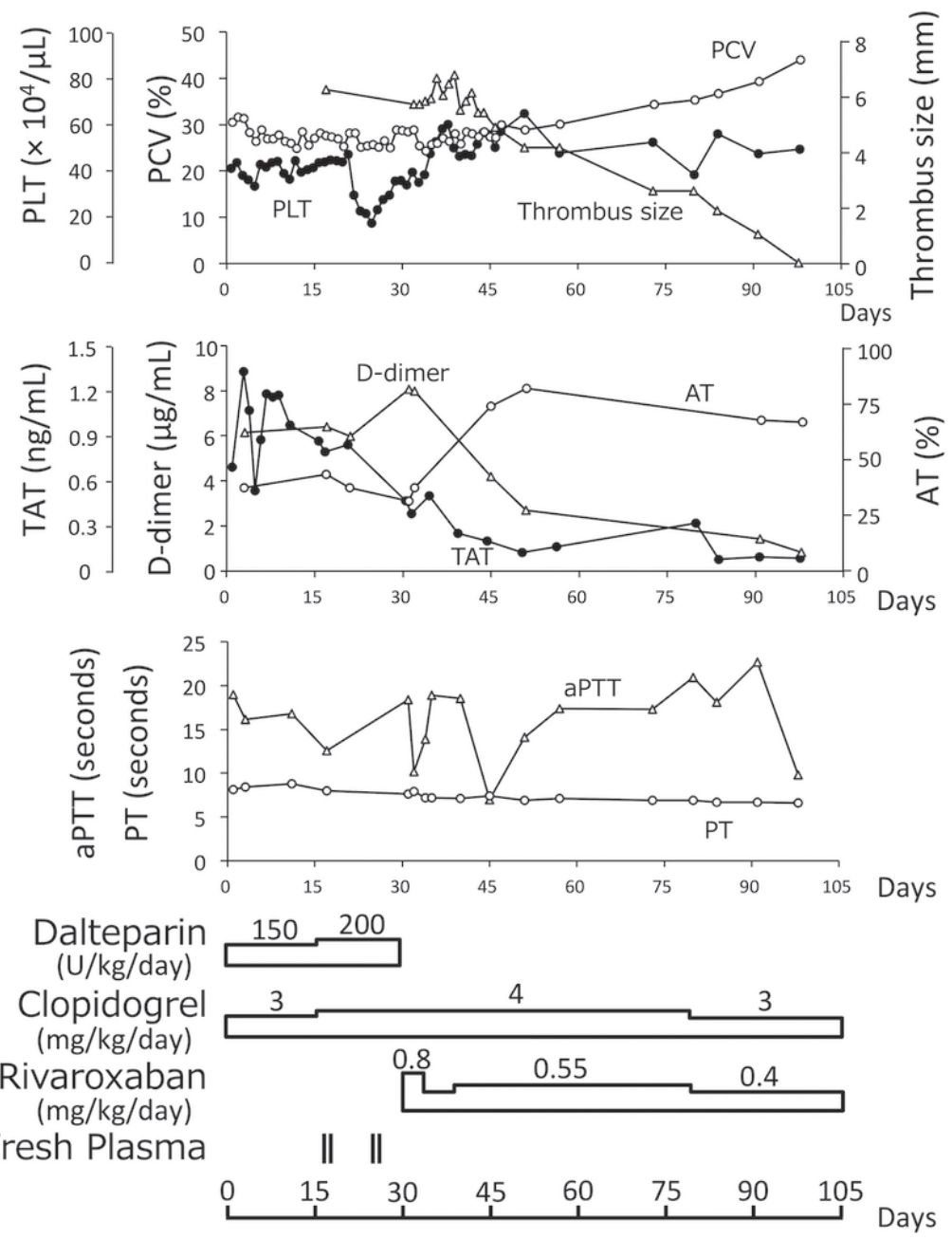

Fig. 2. Treatment and course of disease in Dog 1. Packed cell volume (PCV), the number of platelets (PLT), activated partial thromboplastin time (aPTT), prothrombin time (PT), antithrombin (AT), D-dimer, and thrombin-antithrombin complex (TAT) were measured until 98 days after the first presentation. The maximum short diameter of the thrombus in the splenic vein was measured to indicate the thrombus size by ultrasonography. Names and doses of antithrombotic agents are shown under the graphs of coagulation/fibrinolysis parameters. 


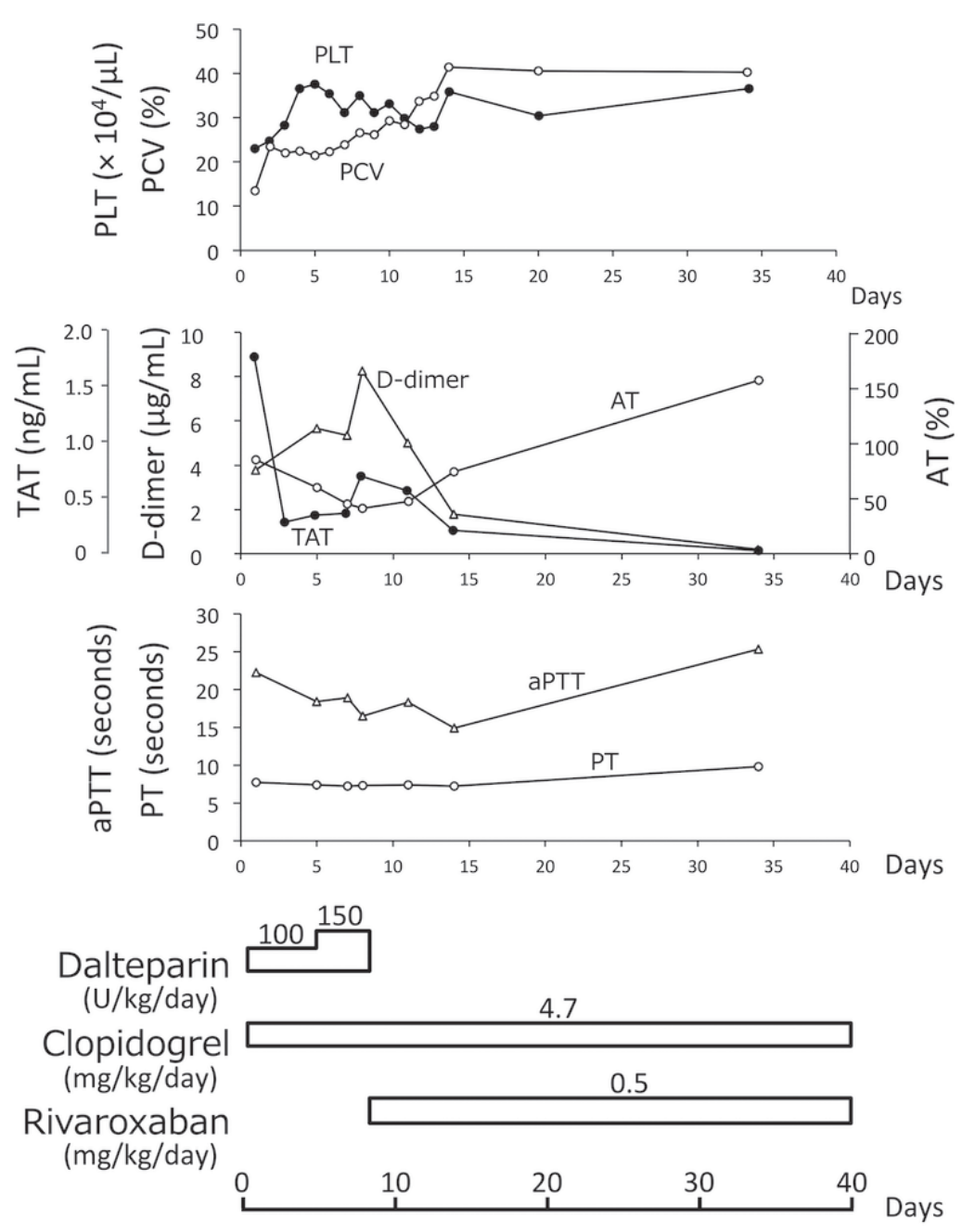

Fig. 3. Treatment and course of disease in Dog 2. Packed cell volume (PCV), the number of platelets (PLT), activated partial thromboplastin time (aPTT), prothrombin time (PT), antithrombin (AT), D-dimer, and thrombin-antithrombin complex (TAT) were measured until 34 days after the first presentation. Names and doses of antithrombotic agents are shown under the graphs of coagulation/fibrinolysis parameters.

the ascites was revealed to be pure transudate by fluid analysis. Although no obvious thrombus was observed, undetectable venous thromboembolism was suspected to be the cause of ascites and edema because of the decreased AT activity (60\%) and increased D-dimer $(8.13 \mu \mathrm{g} / \mathrm{m} l)$ and exclusion of the other diseases such as hypoalbuminemia and congestive heart failure.

Dalteparin and clopidogrel were supposed to be ineffective because there was no improvement of clinical symptom or coagulation profiles, so dalteparin was replaced with rivaroxaban (Xarelto; $0.5 \mathrm{mg} / \mathrm{kg}$, PO, q 24 hr; Bayer Pharma AG) on Day 8. Edema and peritoneal effusion resolved completely on Day 10, and coagulation profiles were also normalized on Day 34 (Fig. 3 ). No evidence of bleeding was observed throughout the course of the disease in this case. aPTT prolonged slightly ( $25.3 \mathrm{sec})$ on Day 34, but still in the reference range. Since no obvious recurrence of thromboembolic symptom was observed by physical examination, ultrasonography and blood examination until Day 50, rivaroxaban was discontinued at this point. Twenty-five days later, clopidogrel was also discontinued because the result of blood examination got improved (PCV: 43.4\%, PT: 6.8 sec, aPTT: $12.7 \mathrm{sec}$, and TAT: $0.028 \mathrm{ng} / \mathrm{m} \mathrm{l}$ ), with no obvious symptoms related to thromboembolism. Until at the time point of manuscript preparation, the dog has kept its good condition for 703 days after cessation of rivaroxaban treatment.

This report introduced the use of rivaroxaban in the treatment of venous thromboembolism secondary to IMHA in two dogs. In both cases, antithrombotic therapy with the combination of dalteparin and clopidogrel was ineffective, but after administration of rivaroxaban, the clinical signs associated with thromboembolism resolved and the coagulation parameters (D-dimer, AT and TAT) normalized.

In this study, thrombi were detectable by imaging examinations in $\operatorname{dog} 1$, but not in $\operatorname{dog} 2$. Treatment with rivaroxaban might be effective for the process of thrombus formation even in the conditions with no apparent thrombus detected by diagnostic imaging. It is conceivable that rivaroxaban could be effective for both of the treatment and prophylaxis of thromboembolism under hypercoagulable state. However, it should be carefully interpreted because the dose of prednisolone was reduced in these cases in parallel with the resolution of IMHA, which may have helped resolution of thromboembolism [5]. 
In two dogs in this report, clinical symptoms and coagulation profiles did not resolve under the treatment with dalteparin; however, they resolved after initiation of rivaroxaban. Heparin requires AT to inactivate factor Xa, while rivaroxaban does not [11], which makes rivaroxaban more effective than heparin in patients with low AT levels [33]. Furthermore, rivaroxaban suppresses thrombin generation more efficiently than heparin by inactivating both factor Xa and factor Xa-prothrombinase-complex [14]. For these reasons, rivaroxaban was considered to be more effective in these cases.

In these two dogs, levels of D-dimer, AT and TAT sensitively paralleled with the disease course of thromboembolism. The two dogs treated in this study showed high D-dimer levels at the first presentation, suggesting the presence of thrombi. They showed reduced AT levels, suggesting the heparin resistance as mentioned above. When thrombin is generated, it rapidly binds with AT and TAT is formed from the early phase of the thrombotic disease process $[4,15,26]$. In the two dogs in this study, TAT concentrations exceeded the reference range, but rapidly decreased soon after resolution of thromboembolic disease. This report supports the utility of D-dimer, AT and TAT for better understanding of the improvement/aggravation and monitoring of thromboembolic diseases in dogs, which supports decision-making on the course of treatment.

In this report, bleeding events associated with the administration of rivaroxaban were observed in Dog 1, although previous studies reported no bleeding events at similar doses $[1,8,22,32]$. It has already been declared that special caution is required in dogs with renal insufficiency, because $52 \%$ of absorbed rivaroxaban is excreted from the kidney in dogs [31]. Considering that neither renal insufficiency detected by blood examination nor ultrasonographic abnormality in the kidney was found at the time of bleeding event in Dog 1, there seemed to be no or minimal damage in the kidney function. Previous report demonstrated individual variation of the pharmacokinetics and response to rivaroxaban in healthy dogs, which may explain the reason for the unexpected bleeding [8]. Considering that bleeding events (mainly minor bleeding events) have been observed in a certain population of human patients treated with rivaroxaban [3], it is reasonable to experience such adverse events in dogs with similar pharmacokinetics and pharmacodynamics of rivaroxaban [1, 8, 30, 31]. Moreover, the dogs in this study were treated with rivaroxaban in combination with clopidogrel, which could have increased the tendency of bleeding. To prevent the bleeding, a major side effect of DOAC, monitoring the anticoagulant efficacy of rivaroxaban is required.

As parameters for anticoagulant effect of rivaroxaban, PT, aPTT and anti-factor Xa activity have been evaluated in humans [2, $10,13,16,23,27,28,34]$, indicating that anti-factor Xa assay is the most suitable for its monitoring. As shown in humans, antifactor Xa assay was shown to be the most sensitive to demonstrate the anticoagulant effect of rivaroxaban in dogs [7]. However, since there are no commercial laboratories to measure anti-factor Xa activity in dogs, other parameters are required. Several studies indicated that PT could be an alternative measurement in practice for the anticoagulant effect of rivaroxaban in humans [25, 27] as well as in dogs [1]. In the present study, the two dogs did not show any prolongation of PT throughout the period of treatment with rivaroxaban. The timing of measurement could be the reason for the lack of PT prolongation in two dogs presented in this study. Since the peak anticoagulant effect of rivaroxaban is observed $1-4 \mathrm{hr}$ from the rivaroxaban administration in dogs [1, 8, 30], investigation at early timing (e. g. $3 \mathrm{hr}$ after administration) is desired to determine a suitable timing for its monitoring in a large number of dog patients. Further investigation is required for establishment of better monitoring methods for safe and effective administration of rivaroxaban in dogs.

This report described the efficacy of rivaroxaban for venous thromboembolism secondary to IMHA in dogs as well as the usefulness of D-dimer, AT, and TAT measurement to monitor the state of thromboembolism. Prospective clinical trials are needed to substantiate the efficacy of rivaroxaban on the treatment of thromboembolism and its monitoring system with D-dimer, AT and TAT in dogs.

\section{REFERENCES}

1. Bae, J., Kim, H., Kim, W., Kim, S., Park, J., Jung, D. I. and Yu, D. 2019. Therapeutic monitoring of rivaroxaban in dogs using thromboelastography and prothrombin time. J. Vet. Intern. Med. 33: 1322-1330. [Medline] [CrossRef]

2. Barrett, Y. C., Wang, Z., Frost, C. and Shenker, A. 2010. Clinical laboratory measurement of direct factor Xa inhibitors: anti-Xa assay is preferable to prothrombin time assay. Thromb. Haemost. 104: 1263-1271. [Medline] [CrossRef]

3. Beyer-Westendorf, J., Förster, K., Pannach, S., Ebertz, F., Gelbricht, V., Thieme, C., Michalski, F., Köhler, C., Werth, S., Sahin, K., Tittl, L., Hänsel, U. and Weiss, N. 2014. Rates, management, and outcome of rivaroxaban bleeding in daily care: results from the Dresden NOAC registry. Blood 124: 955-962. [Medline] [CrossRef]

4. Boisclair, M. D., Lane, D. A., Wilde, J. T., Ireland, H., Preston, F. E. and Ofosu, F. A. 1990. A comparative evaluation of assays for markers of activated coagulation and/or fibrinolysis: thrombin-antithrombin complex, D-dimer and fibrinogen/fibrin fragment E antigen. Br. J. Haematol. 74: 471-479. [Medline] [CrossRef]

5. Brotman, D. J., Girod, J. P., Posch, A., Jani, J. T., Patel, J. V., Gupta, M., Lip, G. Y., Reddy, S. and Kickler, T. S. 2006. Effects of short-term glucocorticoids on hemostatic factors in healthy volunteers. Thromb. Res. 118: 247-252. [Medline] [CrossRef]

6. Breuhl, E. L., Moore, G., Brooks, M. B. and Scott-Moncrieff, J. C. 2009. A prospective study of unfractionated heparin therapy in dogs with primary immune-mediated hemolytic anemia. J. Am. Anim. Hosp. Assoc. 45: 125-133. [Medline] [CrossRef]

7. Conversy, B., Blais, M. C., Dunn, M., Gara-Boivin, C., Carioto, L. and del Castillo, J. R. 2013. Rivaroxaban demonstrates in vitro anticoagulant effects in canine plasma. Vet. J. 198: 437-443. [Medline] [CrossRef]

8. Conversy, B., Blais, M. C., Dunn, M., Gara-Boivin, C. and Del Castillo, J. R. E. 2017. Anticoagulant activity of oral rivaroxaban in healthy dogs. Vet. J. 223: 5-11. [Medline] [CrossRef]

9. de Laforcade, A. M., Freeman, L. M., Shaw, S. P., Brooks, M. B., Rozanski, E. A. and Rush, J. E. 2003. Hemostatic changes in dogs with naturally occurring sepsis. J. Vet. Intern. Med. 17: 674-679. [Medline] [CrossRef]

10. Douxfils, J., Mullier, F., Loosen, C., Chatelain, C., Chatelain, B. and Dogné, J. M. 2012. Assessment of the impact of rivaroxaban on coagulation 
assays: laboratory recommendations for the monitoring of rivaroxaban and review of the literature. Thromb. Res. 130: 956-966. [Medline] [CrossRef]

11. Gulseth, M. P., Michaud, J. and Nutescu, E. A. 2008. Rivaroxaban: an oral direct inhibitor of factor Xa. Am. J. Health Syst. Pharm. 65: 1520-1529. [Medline] [CrossRef]

12. Helmond, S. E., Polzin, D. J., Armstrong, P. J., Finke, M. and Smith, S. A. 2010. Treatment of immune-mediated hemolytic anemia with individually adjusted heparin dosing in dogs. J. Vet. Intern. Med. 24: 597-605. [Medline] [CrossRef]

13. Hillarp, A., Baghaei, F., Fagerberg Blixter, I., Gustafsson, K. M., Stigendal, L., Sten-Linder, M., Strandberg, K. and Lindahl, T. L. 2011. Effects of the oral, direct factor Xa inhibitor rivaroxaban on commonly used coagulation assays. J. Thromb. Haemost. 9: 133-139. [Medline] [CrossRef]

14. Ieko, M. 2014. Characteristics of various anticoaguants and evaluation methods for risk of bleeding and thrombosis. J. Electrocardiol. 34: 149-156. [CrossRef]

15. Kato, D., Takahashi, M., Yonezawa, T., Ohmi, A., Takeda, S., Nakagawa, T., Hosoda, S., Kanemoto, H., Fukushima, K., Ohno, K., Matsuki, N. and Tsujimoto, H. 2020. Evaluation of an automated point-of-care test system for measuring thrombin-antithrombin complex in dogs. J. Vet. Emerg. Crit. Care (San Antonio) 30: 102-106. [Medline] [CrossRef]

16. Kubitza, D., Becka, M., Voith, B., Zuehlsdorf, M. and Wensing, G. 2005. Safety, pharmacodynamics, and pharmacokinetics of single doses of BAY 59-7939, an oral, direct factor Xa inhibitor. Clin. Pharmacol. Ther. 78: 412-421. [Medline] [CrossRef]

17. Kuzi, S., Segev, G., Haruvi, E. and Aroch, I. 2010. Plasma antithrombin activity as a diagnostic and prognostic indicator in dogs: a retrospective study of 149 dogs. J. Vet. Intern. Med. 24: 587-596. [Medline] [CrossRef]

18. Maan, A., Padmanabhan, R., Shaikh, A. Y., Mansour, M., Ruskin, J. N. and Heist, E. K. 2012. Newer anticoagulants in cardiovascular disease: a systematic review of the literature. Cardiol. Rev. 20: 209-221. [Medline] [CrossRef]

19. Marschner, C. B., Kristensen, A. T., Rozanski, E. A., McEvoy, F. J., Kühnel, L., Taeymans, O., de Laforcade, A., Sato, A. F. and Wiinberg, B. 2017. Diagnosis of canine pulmonary thromboembolism by computed tomography and mathematical modelling using haemostatic and inflammatory variables. Vet. J. 229: 6-12. [Medline] [CrossRef]

20. Maruyama, H., Watari, T., Miura, T., Sakai, M., Takahashi, T., Koie, H., Yamaya, Y., Asano, K., Edamura, K., Sato, T., Tanaka, S., Hasegawa, A. and Tokuriki, M. 2005. Plasma thrombin-antithrombin complex concentrations in dogs with malignant tumours. Vet. Rec. 156: 839-840. [Medline] [CrossRef]

21. Mellett, A. M., Nakamura, R. K. and Bianco, D. 2011. A prospective study of clopidogrel therapy in dogs with primary immune-mediated hemolytic anemia. J. Vet. Intern. Med. 25: 71-75. [Medline] [CrossRef]

22. Morassi, A., Bianco, D., Park, E., Nakamura, R. K. and White, G. A. 2016. Evaluation of the safety and tolerability of rivaroxaban in dogs with presumed primary immune-mediated hemolytic anemia. J. Vet. Emerg. Crit. Care (San Antonio) 26: 488-494. [Medline] [CrossRef]

23. Okata, T., Toyoda, K., Okamoto, A., Miyata, T., Nagatsuka, K. and Minematsu, K. 2014. Anticoagulation intensity of rivaroxaban for stroke patients at a special low dosage in Japan. PLoS One 9: e113641. [Medline] [CrossRef]

24. Panek, C. M., Nakamura, R. K. and Bianco, D. 2015. Use of enoxaparin in dogs with primary immune-mediated hemolytic anemia: 21 cases. J. Vet. Emerg. Crit. Care (San Antonio) 25: 273-277. [Medline] [CrossRef]

25. Perzborn, E., Strassburger, J., Wilmen, A., Pohlmann, J., Roehrig, S., Schlemmer, K. H. and Straub, A. 2005. In vitro and in vivo studies of the novel antithrombotic agent BAY 59-7939-an oral, direct Factor Xa inhibitor. J. Thromb. Haemost. 3: 514-521. [Medline] [CrossRef]

26. Rimpo, K., Tanaka, A., Ukai, M., Ishikawa, Y., Hirabayashi, M. and Shoyama, T. 2018. Thrombin-antithrombin complex measurement using a point-of-care testing device for diagnosis of disseminated intravascular coagulation in dogs. PLoS One 13: e0205511. [Medline] [CrossRef]

27. Samama, M. M., Martinoli, J. L., LeFlem, L., Guinet, C., Plu-Bureau, G., Depasse, F. and Perzborn, E. 2010. Assessment of laboratory assays to measure rivaroxaban — an oral, direct factor Xa inhibitor. Thromb. Haemost. 103: 815-825. [Medline] [CrossRef]

28. Samuelson, B. T., Cuker, A., Siegal, D. M., Crowther, M. and Garcia, D. A. 2017. Laboratory assessment of the anticoagulant activity of direct oral anticoagulants: A systematic review. Chest 151: 127-138. [Medline] [CrossRef]

29. Swann, J. W., Garden, O. A., Fellman, C. L., Glanemann, B., Goggs, R., LeVine, D. N., Mackin, A. J. and Whitley, N. T. 2019. ACVIM consensus statement on the treatment of immune-mediated hemolytic anemia in dogs. J. Vet. Intern. Med. 33: 1141-1172. [Medline] [CrossRef]

30. Weinz, C., Buetehorn, U., Daehler, H. P., Kohlsdorfer, C., Pleiss, U., Sandmann, S., Schlemmer, K. H., Schwarz, T. and Steinke, W. 2005. Pharmacokinetics of BAY 59-7939 - an oral, direct Factor Xa inhibitor — in rats and dogs. Xenobiotica 35: 891-910. [Medline] [CrossRef]

31. Weinz, C., Schwarz, T., Kubitza, D., Mueck, W. and Lang, D. 2009. Metabolism and excretion of rivaroxaban, an oral, direct factor Xa inhibitor, in rats, dogs, and humans. Drug Metab. Dispos. 37: 1056-1064. [Medline] [CrossRef]

32. Yang, V. K., Cunningham, S. M., Rush, J. E. and de Laforcade, A. 2016. The use of rivaroxaban for the treatment of thrombotic complications in four dogs. J. Vet. Emerg. Crit. Care (San Antonio) 26: 729-736. [Medline] [CrossRef]

33. Zhang, L., Zhang, H., Zhang, J., Tian, H., Liang, J. and Liu, Z. 2018. Rivaroxaban for the treatment of venous thromboembolism in patients with nephrotic syndrome and low AT-III: A pilot study. Exp. Ther. Med. 15: 739-744. [Medline]

34. Zhang, Y., Qian, Q., Qian, G. and Sun, G. 2016. Laboratory monitoring of rivaroxaban and assessment of its bleeding risk. Br. J. Biomed. Sci. 73: 134-139. [Medline] [CrossRef] 